\title{
Potentila indica (Andr.) nov. (wild strawberry) in Sri Lanka is Restricted to a Small Climatic Envelop Urging Strict Conservation
}

\author{
L.T. Ranaweera ${ }^{1}$, H.A.C.R. Perera ${ }^{1,2}$, W.W.M.U.K. Wijesundara ${ }^{1}$, R.M.S.K. Rathnayake ${ }^{1}$, \\ W.M.D.A. Wijesundara ${ }^{1}$, H.M.T.N. Senavirathna ${ }^{1}$, R.W.K.M. Senevirathna ${ }^{1}$, C.K. Weebadde ${ }^{3}$, \\ J.F. Hancock ${ }^{4}$ and S.D.S.S. Sooriyapathirana ${ }^{1,2^{*}}$
}

Date Received: $26^{\text {th }}$ February 2020 / Date Accepted: $14^{\text {th }}$ August 2020

\begin{abstract}
Purpose : The nomenclature of the wild strawberries inhabited in Sri Lanka is ambiguous. In Sri Lanka, this species is still named Duchesnea indica which needs a revision. Wild strawberries grow well in natural habitats of upcountry in Sri Lanka. Since the commercial strawberry cultivations gain a popularity in upcountry, the studies on wild strawberry is essential for crop improvement and management.

Research Method: In the present study, we conducted extensive field sampling followed by a phylogenetic analysis with the DNA barcoding markers ITS and, trnL-F by using a representative sample of wild strawberry plants in Sri Lanka. The distribution of the species was identified using maximum entropy modeling approaches.

Findings : Sri Lankan wild strawberry got placed at subtribe: Potentilla, and clade: Reptans and show a shallow divergence with the species Potentilla indica reported. Thus, we reposition the genus of wild strawberries in Sri Lanka from Duchesnea to Potentilla and hereafter name it as P. indica. The niche model analysis predicted a highly restricted distribution of Sri Lankan wild strawberry in Nuwara-Eliya district over an area of $166.36 \mathrm{~km}^{2}$ in the altitude range of $1546-2524 \mathrm{~m}$ in a small climatic envelop highlighting the need for urgent conservation measures.
\end{abstract}

Research Limitations : The pop-set for available in literature of P. indica is limited for comparison. Extensive studies based on DNA sequencing is needed for further validation.

Originality / Value : Taxonomy, narrow distribution, need of conservation, and phylogenetic distance to Fragaria chiloensis, a progenitor species of cultivated strawberry, are defined for Sri Lankan wild strawberries.

Keywords: Duchesnea indica, ecological niche modeling of Potentilla, Indian strawberry, Reptans, wild strawberry in Sri Lanka

\section{INTRODUCTION}

In Sri Lanka, the wild strawberries (also known as Indian or mock strawberries) are named under the genus Duchesnea (Focke, 1888) in the Revised Handbook to the Flora of Ceylon (Wadhwa et al., 2000). The genus of the wild strawberries in Sri Lanka was later changed to Fragaria (Andrews, 1807) and then to Potentilla (Wolf, 1908). However, later the taxonomists have agreed to keep wild strawberries in Sri Lanka in the genus Duchesnea which is considered more of a transitional group between Fragaria and
1 Department of Molecular Biology and Biotechnology, Faculty of Science, University of Peradeniya, 20400, Peradeniya, Sri Lanka.

sunethss09@gmail.com

Postgraduate Institute of Science, University of Peradeniya, Sri Lanka.

3 Department of Plant, Soil and Microbial Sciences, Michigan State University, East Lansing, Michigan, USA.

4 Department of Horticulture, Michigan State University, East Lansing, Michigan, USA.

(D)http://orcid.org/0000-0002-5592-1742 
Potentilla (Wadhwa et al., 2000). However, the current nomenclature of wild strawberries in Sri Lanka (Duchesnea indica) is solely dependent on morphology, and no molecular systematic studies were conducted to support the taxonomic status reported in Revised Hanbook to the Flora of Ceylon (Wadhwa et al., 2000).

Potentilleae is one of the taxonomically ambiguous tribes in family Rosaceae. The taxonomy of Potentilleae has been consistently changing over the recent years in world-wide studies (Feng et al., 2017). In the study conducted by Eriksson et al. (1998), described Potentilla as a non-monophyletic genus, combining previously recognized genera such as Duchesnea, Horkelia and Ivesia under Potentilla. The most recent systematic studies using molecular data (Potter et al., 2007; Dobeš and Paule, 2010; Töpel et al., 2011) support the monophyly of the tribe Potentilleae as defined by a study done by Eriksson et al. (2003) and have recovered three main clades within this tribe. Töpel et al. (2011) compared a nuclear and chloroplast gene-based tree to identify topological incongruences that may indicate the hybridization events within the genus Potentilla. The recent phylogenetic studies on Potentilleae were carried out using combined analysis of chloroplast and nuclear markers to further resolve the taxonomic ambiguities (Wadhwa et al., 2000). Once the taxonomic ambiguities are resolved, it is essential to assess and demarcate the conservation priorities of the available germplasm.

The prediction of the ecological distribution of a species is one of the critical aspects when setting up the conservation priorities for a species (Faith, 1996; Geneletti, 2004). The occurrence of a species can be modeled and predicted using an array of methods (Peterson, 2003; Wiens and Graham, 2005; Elith et al., 2006; Kozak et al., 2008; Peterson et al., 2011). The niche modeling using the species-occurrence/location data is popular as the locations of presence are well documented or easy to record for a particular species (Elith et al., 2006; Hernandez et al., 2006; Phillips and Dudík, 2008; Wisz et al., 2008). In the model calibration, we can use presencelocations and set of environmental variables. However, when using the presence-locations of species, some areas could be oversampled or under-sampled leaving a bias (Hortal et al., 2008; Anderson and Gonzalez, 2011). Thus the over-fitting of the models could happen when the model is incorporated with environmental variables (Anderson and Gonzalez, 2011). Another common problem is the over-fitting of model to noise when the number of predictor variables become too high. This confounds the ultimate niche of the species from the predicted niche. Such issues can be avoided using maximum entropy modeling methods (Phillips et al., 2004; Phillips et al., 2006). Through identification of the niche of a species; the distribution pattern, as well as the climatic factors supporting the distribution can be tested (Geneletti, 2004). Currently distribution modeling is used as a versatile tool to identify the populations and species that are climatically restricted to device the conservation priorities (Senevirathne et al., 2018; Wijayathilaka et al., 2018).

In the present study, we aimed to determine the phylogenetic position of wild strawberries inhabited in Sri Lanka. Since the morphology of wild strawberries has been well described, we conducted a molecular systematic analysis using nuclear genome specific ITS and chloroplast genome specific trnL-trnF markers to find the correct phylogenetic position in comparison to other species in the tribe Potentilleae. Then we conducted an extensive field survey to determine the distribution of wild strawberries to get an understanding of the niche dimensions using maximum entropy modeling and introduce conservational priorities.

\section{MATERIALS AND METHODS}

\section{Field Survey and Sample collection}

The species range and the habitat information were collected from the revised handbook to the Flora of Ceylon [Wadhwa et al., 2000] and Royal Botanical Garden, Peradeniya, Sri Lanka. According to the previous species occurrence records, the species was concentrated on central hills of Sri Lanka. Thus, numerous field surveys were conducted across the central hills in Sri Lanka. Once the species was found in a particular location, the GPS coordinates were recorded, and three to five leaf samples were collected. The 
collected leaf samples were stored in $-20^{\circ} \mathrm{C}$ until the DNA extraction.

\section{PCR and DNA sequencing}

The DNA sequencing was performed for two DNA markers namely ITS (ITS1T C C G T A G G T G A A C C T T G C G G, ITS 4 - T C C T C C GC T TAT T GATAT GC) (White et al., 1990) and trnL-trnF (F-CGAAATCGGTAGACGCTACG, $R$ ATTTGAACTGGTGACACGAG) (Taberlet et al., 1991). The PCR was carried out in $50 \mu \mathrm{L}$ of total reaction mixture comprised of $25 \mu \mathrm{L}$ of Go Taq ${ }^{\circledR}$ Green Master Mix, $2 \times$ Green Go Taq ${ }^{\circledR}$ Reaction Buffer (pH 8.5), $400 \mu \mathrm{L}$ dATP, $400 \mu \mathrm{L}$ dGTP, $400 \mu \mathrm{L}$ dCTP, $400 \mu \mathrm{L}$ dTTP, $3 \mathrm{mM} \mathrm{MgCl}, 1$ $\mu \mathrm{L}$ of forward primer, $1 \mu \mathrm{L}$ of reverse primer, template DNA, and nuclease free water. The PCR cyclic process conducted in the Thermal Cycler (Takara, Otsu Shiga, Japan) was consisted of initial denaturation at $94{ }^{\circ} \mathrm{C}$ for 5 mins, followed by 35 cycles including $30 \mathrm{sec}$ of denaturation at $94{ }^{\circ} \mathrm{C}, 1 \mathrm{~min}$ annealing at $55{ }^{\circ} \mathrm{C}$ and 2 mins extension at $72^{\circ} \mathrm{C}$ followed by final extension of 10 mins at $72{ }^{\circ} \mathrm{C}$ (Senavirathna et al., 2020). The PCR products were visualized using $1 \%$ agarose gel electrophoresis and purified using QIAquick PCR purification kit (Qiagen, Hilden, Germany). The purified products were subjected to cycle sequencing using ABI Genetic analyzer 3500 (Applied Bio Systems ${ }^{\circledR}$ ).

\section{Phylogenetic analysis}

The raw sequence reads were edited, and consensus sequences were constructed using the MEGA software v.7 (Kumar et al., 2016). All the consensus sequences generated in the present study were submitted to the GenBank under the accession numbers MK605458-MK60573 and MK587724-MK587739. To examine the precise phylogenetic position of the study species, we adapted the phylogeny constructed in a study by Feng et al. (2017) (Table 01) using both nuclear and plastid genetic markers. We attempted to reconstruct the tribe: Potentilleae phylogeny using the sequences generated in the present study and sequences reported in previous studies (Feng et al., 2017). We constructed the multiple sequence alignments separately for ITS and trnL-trnF in MEGA v7. A partition homogeneity (ILD) test (Planet, 2006) was carried out to check the phylogenetic concordance of ITS, trnL-trnF, and ITS+trnL-trnF (combined) datasets. To define the best partition scheme and the best model of evolution for each data matrix, we implemented the analysis in PartitionFinder 2 (Lanfear et al., 2016). In PartitionFinder 2, we implemented the corrected Akaike information criteria (Cavanaugh, 1997) (AICc) using hcluster (Lanfear et al., 2014) and Kmeans (Frandsen et al., 2015) algorithms for model selection.

We carried out the tree search in both Maximum Likelihood (ML) and Bayesian frameworks. The ML tree search was implemented using the rapid bootstrap algorithm (Stamatakis et al., 2008) for 1000 iterations in RAxML-VI-HPC workflow (Stamatakis, 2006) using CIPRES supercomputer (Miller et al., 2010). We used the GTRGAMMA model to assess the evolutionary process of the partitions defined in the analysis. Using the bipartition option of the RAxML, we performed a consensus algorithm to conclude all the bootstrap bipartitions into a single tree topology. The best tree resulted with highest $-\log$ likelihood value was used as the tree topology to imprint the bootstrap values. We also constructed a phylogenetic tree based on the Bayesian framework by employing MrBays (Huelsenbeck and Ronquist, 2001) in the CIPRES platform (Miller et al., 2010). During this tree run, differences were assessed separately, which were achieved by the partition log file that contains the model information and partition criteria. Two hot and cold chains of Markov chain Monte Carlo (MCMC) were run for 60 million generations to probe the trees in tree-space. The final 50\% majority rule consensus tree was drawn using the trees probed after maximum chain convergence. However, the initial $25 \%$ of the trees were discarded as burn-in. The chain convergence and independent tree sampling were checked by assessing Effective Sample Size (ESS) in TRACER v1.4 (Rambaut and Drummond, 2007). Finally, all the trees constructed in the study were visualized and edited using FigTree v1.4.3 (Rambaut, 2014). 
Table 01: The metadata of the sequences used for the phylogenetic analysis

\begin{tabular}{|c|c|c|c|c|}
\hline \multirow{2}{*}{ Taxa } & \multirow{2}{*}{ Voucher } & \multicolumn{2}{|c|}{ Accession Number } & \multirow{2}{*}{ Reference } \\
\hline & & ITS & trnL/trnF & \\
\hline Potentilla indica & DMB61 & MK587724 & $M K 605458$ & \multirow{16}{*}{ This study } \\
\hline Potentilla indica & DMB62 & MK587725 & MK605459 & \\
\hline Potentilla indica & DMB63 & MK587726 & MK605460 & \\
\hline Potentilla indica & DMB64 & MK587727 & MK605461 & \\
\hline Potentilla indica & DMB65 & MK587728 & MK605462 & \\
\hline Potentilla indica & DMB66 & MK587729 & MK605463 & \\
\hline Potentilla indica & DMB67 & MK587730 & MK605464 & \\
\hline Potentilla indica & DMB68 & MK587731 & MK605465 & \\
\hline Potentilla indica & DMB69 & MK587732 & MK605466 & \\
\hline Potentilla indica & DMB70 & MK587733 & MK605467 & \\
\hline Potentilla indica & DMB71 & MK587734 & MK605468 & \\
\hline Potentilla indica & DMB72 & MK587735 & MK605469 & \\
\hline Potentilla indica & DMB73 & MK587736 & MK605470 & \\
\hline Potentilla indica & DMB74 & MK587737 & MK605471 & \\
\hline Potentilla indica & DMB75 & MK587738 & MK605472 & \\
\hline Potentilla indica & DMB76 & MK587739 & MK605473 & \\
\hline Alchemilla_cryptantha_Steud._ex_A._Rich & T._Eriksson_914_(S) & FJ356153 & FJ422283 & \multirow{30}{*}{$\begin{array}{l}\text { Feng et al. } \\
\quad \text { (2017) }\end{array}$} \\
\hline Alchemilla_mollis_(Buser)_Rothm. & T._Eriksson_s.n.(S) & AJ511769 & AJ512218 & \\
\hline Alchemilla_pentaphyllea_L. & B._Gehrke_BG-E400_(ZH) & FJ356154 & FJ422284 & \\
\hline Argentina_anserina_(L.)_Rydb. & $\begin{array}{l}\text { Eriksson_\&_Smedmark_44_ } \\
\text { (SBT) }\end{array}$ & FN430824 & FN561752 & \\
\hline $\begin{array}{l}\text { Argentina_glabriuscula_(T.T. } \\
\mathrm{Y} € \mathrm{u} \text { _\&_C.L.Li)_Sojak_53 }\end{array}$ & Feng_53_(HIB) & KF954763 & KJ020639 & \\
\hline $\begin{array}{l}\text { Argentina_glabriuscula_(.T. } \\
\mathrm{Y} € \mathrm{u} \& \text { C.L.Li) Sojak } 57\end{array}$ & Feng_57_(HIB) & KF954764 & KJ020640 & \\
\hline Argentina_leuconota_(D.DDon T)_Sojak & Feng_108_(HIB) & KF954771 & KJ020641 & \\
\hline $\begin{array}{l}\text { Argentina_lignosa_(Willd. } \\
\text { in_D.F.K.Schltdl.)_Sojak }\end{array}$ & M._Topel_MA132_(GB) & FJ356171 & FJ422299 & \\
\hline Argentina_micropetala_(D._Don)_Sojak & Feng_8_(HIB) & KF954771 & KJ020641 & \\
\hline Argentina_microphylla_(D._Don)_Sojak & MA_144_(GB) & FN430809 & FN556412 & \\
\hline Argentina_peduncularis_(D._Don)_Sojak & MA_173_(GB) & FN430820 & FN561742 & \\
\hline $\begin{array}{l}\text { Argentina_phanerophlebia_(Yu_\&_Li)_ } \\
\text { Feng_\&_Wang }\end{array}$ & Feng_6_(HIB) & KF954770 & KJ020642 & \\
\hline Argentina_songzhuensis_T. Feng_\&_H.Wang & Feng_58_(HIB) & KF954766 & KJ020638 & \\
\hline Argentina_stenophylla_(Franch.)_Sojak & KGB_299_(GB) & FN555607 & FN561738 & \\
\hline Argentina_tapetodes_(Sojak)_Sojak & Feng_93_(HIB) & KF954769 & KP875330 & \\
\hline Argentina_turfosa_(Hand.-Mazz.)_Sojak & Feng_55_(HIB) & KF954768 & KP875331 & \\
\hline $\begin{array}{l}\text { Chamaecallis_perpusilloides_(W.W._Sm.) } \\
\text { Smedmark }\end{array}$ & Feng_52_(HIB) & KP875287 & KP875336 & \\
\hline $\begin{array}{l}\text { Chamaecallis_perpusilloides_(W.W._Sm.) } \\
\text { Smedmark }\end{array}$ & Feng_68_(HIB) & KP875288 & KP875335 & \\
\hline Chamaerhodos_mongholica_Bunge & E._Rosenius_1028_(S) & FJ356155 & FJ422285 & \\
\hline Dasiphora_davurica_(Nestl.)_Kom._\&_Aliss & M._Lundberg_24_(S) & FJ356159 & FJ422287 & \\
\hline Dasiphora_fruticosa_(L.)_Rydb. & Feng_103_(HIB) & KP875290 & KP875337 & \\
\hline Dasiphora_glabra_(G._Lodd.)_Sojak & Feng_120_(HIB) & KP875289 & KP875338 & \\
\hline Dasiphora_parvifolia(Fisch._ex_Lehm.)_Juz. & Feng_119_(HIB) & KF954762 & KJ020646 & \\
\hline Dasiphora_phyllocalyx_Juz. & T._Eriksson_757_(S) & FJ356160 & FJ422288 & \\
\hline $\begin{array}{l}\text { Drymocallis_corsica_(Soleirol_ex_Lehm.) } \\
\text { Kurtto }\end{array}$ & M._Lundberg_13_(S) & FJ356161 & FJ422290 & \\
\hline Drymocallis_glutinosa_Rydb. & M._Lundberg_5_(S) & FJ356162 & FJ42229 & \\
\hline Drymocallis_rupestris_(L.)_Sojak & M._Lundberg_6_(S) & FJ356163 & FJ422292 & \\
\hline Fragaria_chiloensis_(L.)_Mill. & M._Lundberg_14_(S) & FJ356164 & FJ422293 & \\
\hline Fragaria_orientalis_Losinsk. & Feng_107_(HIB) & KP875292 & KР875334 & \\
\hline Fragaria_viridis_Weston & M._Lundber_16_(S) & FJ356166 & FJ422295 & \\
\hline
\end{tabular}


Horkelia_bolanderi_A._Gray

Ivesia_kingii_S._Watson

Potaninia_mongolica_Maxim

Potentilla_alba_L.

Potentilla_alchemilloides_Lapeyr.

Potentilla_argentea_L.

Potentilla_biflora_Willd._ex_Schltdl._102

Potentilla_caulescens_L.

Potentilla_chinensis_Ser.

Potentilla_clandestina_Sojak

Potentilla_conferta_Bunge

Potentilla_discolor Bunge

Potentilla_griffithii_Hook._f.

Potentilla_indica_(Andrews)_Wolf

Potentilla_kleiniana_Wight_\&_Arn.

Potentilla_multifida_(Tausch)_Wolf

Potentilla_purpurea_(Royle)_Hook._f.

Potentilla_reptans_L.

Potentilla_sischanensis_Bunge_ex_Lehm.

Potentilla_stolonifera_Lehm._ex_Ledeb

Potentilla_suavis_Sojak

Potentilla_tenuis_(Hand-Mazz.)_Sojak

Potentilla_tetrandra_(Hook._f.)_Bunge_89

Potentilla_tetrandra_(Hook._f.)_Bunge_97

Rosa_majalis_Herrm.

Sibbaldia_parviflora_Willd.

Sibbaldia_procumbens_L.

Sibbaldia_procumbens_L

Sibbaldia_semiglabra_C._A._Mey.

Sibbaldianthe_adpressa_(Bunge)_Juz.G11

Sibbaldianthe_sericea_Grubov

Sibbaldiopsis_cuneifolia_(Bertol.)_Sojak_G5

Sibbaldiopsis_cuneifolia_(Bertol.)_Sojak_48

Eriksson_s.n._(SBT)
J._L._Reveal_et_al.\#4782_
(GB)
Norlindh_\&_Ahti_10348_(S)
MA_122_(GB)
A._\&_A-L._Anderberg_26_
(S)
MA_143_(GB)
Feng_102_(HIB)
MA_133_(GB)
Feng_110_(HIB)
Feng_25_(HIB)
Feng_127_(HIB)
Feng_118_(HIB)
Feng_44_(HIB)
Feng_138_(HIB)
Feng_139_(HIB)
Feng_124_(HIB)
Feng_64_(HIB)
MA_131_(GB)
Feng_112_(HIB)
BE_1382:_1_(GB)
Feng_37_(HIB)
Feng_26_(HIB)
Feng_89_(HIB)
Feng_97_(HIB)
T._Eriksson_641_(GH,_S)
M._Lundberg_4_(S)
Feng_131_(HIB)
Feng_S4_(HIB)
J.Klackenberg82062-11_(S)
V.A._Gusev_391_(S)
Feng_122_(HIB)
M._Lundberg_39_(S)
Feng_48_(HIB)

FN430789

FN556395

FN430787 FN561735

AM286742 AM286743

FN430774 FN556379

FJ356168 FJ422297

FN430808 FN561750

KP875301 KP875329

FN430819 FN556399

KP875298 KP875319

KP875308 KP875327

KP875296 KP875320

KP875299 KP875321

KP875293 KP875316

KP875300 KP875314

KP875294 KP875315

KP875295 KP875318

KP875307 KP875326

FN430815 FN561728

KP875297 KP875322

FN430814 FN556420

KP875305 KP875323

KP875306 KP875325

KP875303 KP875328

KP875304 KP875324

U90801 AJ512229

FJ356174 FJ422302

KP875310 KP875339

KP875309 KP875341

FJ356175 FJ422303

FJ356176 FJ422304

KP875312 KP875333

FJ356169 FJ422298

KP875313 KP875340
Feng et al. (2017)

\section{Ecological niche modeling (ENM)}

We modeled the predicted niche of wild strawberries in Sri Lanka using maximum entropy modeling approach methods (Phillips et al., 2004; Phillips et al., 2006). We used Maxent version 3.3.3 k program (Phillips et al., 2006) to model the species distribution. Maxent only uses presence-only data for entropy modeling. Thus we used 162 presence-localities taken during our field visits (Table 02). We also used 19 bioclimatic variables as the environmental layers for our analysis (Table 03). Maxent often deals with the model overfitting errors, thus to obtain optimum maxent model, we used "species-specific tuning" approach (Anderson and Gonzalez, 2011; Elith et al., 2011). We smoothened our model by choosing different feature classes [Auto, Linear (L), Quadratic (Q), Product (P), Threshold
(T), Hing $(\mathrm{H})]$ between different $\beta$ regulation parameters $(0.01,0.1,0.5,1,2,3,5)$. The model performance evaluated under three approaches. Initially, we assessed the model performance using threshold dependent manner, where we implemented different feature classes to achieve the best-tuned model. The linear and quadratic features are more informative when the number of occurrence points is 20 or less (Anderson and Gonzalez, 2011). Thus we evaluated our model using $\mathrm{P}, \mathrm{T}, \mathrm{H}, \mathrm{L}+\mathrm{Q}+\mathrm{P}, \mathrm{L}+\mathrm{Q}+\mathrm{P}+\mathrm{T}$, and $\mathrm{L}+\mathrm{Q}+\mathrm{P}+\mathrm{T}+\mathrm{H}$. Then we implemented the threshold-independence of the method by assessing Area Under Curve (AUC) value of the Receiver Operation Curves (ROC). Finally, we visualized the model performance by checking the graphical outputs generated during the model building. We ran the program for 5000 iterations, and variable importance was measured using 
jackknifing. We set $10 \%$ of the occurrence data as the test data and kept other parameters as default. The best model was further edited in ArcGIS v10.4. A $20 \%$ threshold was set for the species distribution. We overlaid predicted species distribution into a 3-D map in ArcScene v 10.4 using 30-meter Digital Elevation Map (DEM) profile available in Jet Propulsion Laboratory (JPL) of California Institute of Technology. Finally, we used Google Earth Pro software to overlap our predicted niche with high-resolution satellite image of the predicted niche.

Table 02: The geographical locations of the Potentilla indica presence recorded during this study

\begin{tabular}{|c|c|c|c|}
\hline Sample & Longitude & Latitude & Location \\
\hline $\mathrm{R} 1$ & 7.084561 & 80.724723 & \multirow{12}{*}{ Chariot path mountain } \\
\hline $\mathrm{R} 2$ & 7.083933 & 80.722792 & \\
\hline $\mathrm{R} 3$ & 7.082719 & 80.724079 & \\
\hline $\mathrm{R} 4$ & 7.082442 & 80.726257 & \\
\hline R5 & 7.081324 & 80.731707 & \\
\hline R6 & 7.083134 & 80.738509 & \\
\hline R7 & 7.083847 & 80.736599 & \\
\hline $\mathrm{R} 8$ & 7.083293 & 80.739592 & \\
\hline $\mathrm{R} 9$ & 7.083229 & 80.741223 & \\
\hline $\mathrm{R} 10$ & 7.082249 & 80.743101 & \\
\hline R11 & 7.081408 & 80.740912 & \\
\hline $\mathrm{R} 12$ & 7.081163 & 80.73823 & \\
\hline $\mathrm{R} 13$ & 7.02571 & 80.762499 & \multirow{11}{*}{ Piduruthalagala mountain } \\
\hline $\mathrm{R} 14$ & 7.02703 & 80.770513 & \\
\hline $\mathrm{R} 15$ & 7.019342 & 80.77489 & \\
\hline $\mathrm{R} 16$ & 7.017372 & 80.786584 & \\
\hline R17 & 7.006777 & 80.786938 & \\
\hline $\mathrm{R} 18$ & 7.006777 & 80.786938 & \\
\hline R19 & 7.006553 & 80.789223 & \\
\hline $\mathrm{R} 20$ & 6.999376 & 80.787603 & \\
\hline $\mathrm{R} 21$ & 6.993445 & 80.790425 & \\
\hline $\mathrm{R} 22$ & 6.990767 & 80.789787 & \\
\hline $\mathrm{R} 23$ & 6.999307 & 80.771398 & \\
\hline R24 (DMB71) & 6.942364 & 80.715941 & \multirow{12}{*}{ Radella } \\
\hline $\mathrm{R} 25$ & 6.941901 & 80.716665 & \\
\hline R26 (DMB72) & 6.941017 & 80.715528 & \\
\hline $\mathrm{R} 27$ & 6.939611 & 80.719358 & \\
\hline R28 (DMB73) & 6.938706 & 80.716429 & \\
\hline $\mathrm{R} 29$ & 6.938812 & 80.714648 & \\
\hline $\mathrm{R} 30$ & 6.933966 & 80.716322 & \\
\hline R31 & 6.93338 & 80.716499 & \\
\hline $\mathrm{R} 32$ & 6.932342 & 80.716617 & \\
\hline R33 & 6.931799 & 80.716306 & \\
\hline R34 & 6.931022 & 80.716703 & \\
\hline $\mathrm{R} 35$ & 6.930287 & 80.718817 & \\
\hline $\mathrm{R} 36$ & 6.93158 & 80.718412 & \multirow{6}{*}{ Radella (Black Pool junction) } \\
\hline R37 & 6.929439 & 80.719356 & \\
\hline R38 (DMB74) & 6.926803 & 80.720761 & \\
\hline R39 & 6.925184 & 80.719774 & \\
\hline $\mathrm{R} 40$ & 6.924183 & 80.72031 & \\
\hline R41 & 6.924023 & 80.720664 & \\
\hline
\end{tabular}




\begin{tabular}{|c|c|c|c|}
\hline $\mathrm{R} 42$ & 6.921446 & 80.725889 & \multirow{9}{*}{ Radella (Black Pool junction) } \\
\hline R43 (DMB75) & 6.919348 & 80.729494 & \\
\hline R44 & 6.915418 & 80.734451 & \\
\hline $\mathrm{R} 45$ & 6.912297 & 80.739118 & \\
\hline R46 & 6.915737 & 80.739762 & \\
\hline $\mathrm{R} 47$ & 6.922575 & 80.735857 & \\
\hline R48 (DMB76) & 6.932017 & 80.737423 & \\
\hline R49 & 6.939259 & 80.742659 & \\
\hline $\mathrm{R} 50$ & 6.949238 & 80.771069 & \\
\hline R51 (DMB66) & 6.948583 & 80.775189 & \multirow{40}{*}{ Meepilimana } \\
\hline $\mathrm{R} 52$ & 6.947699 & 80.774417 & \\
\hline $\mathrm{R} 53$ & 6.946613 & 80.773612 & \\
\hline R54 & 6.944105 & 80.775575 & \\
\hline R55 & 6.944243 & 80.776487 & \\
\hline R56 & 6.943892 & 80.777485 & \\
\hline R57 (DMB67) & 6.94304 & 80.778021 & \\
\hline $\mathrm{R} 58$ & 6.942508 & 80.777624 & \\
\hline R59 & 6.941965 & 80.776605 & \\
\hline R60 & 6.941454 & 80.775747 & \\
\hline R61 & 6.940677 & 80.77609 & \\
\hline R62 & 6.939761 & 80.774749 & \\
\hline R63 & 6.937705 & 80.774449 & \\
\hline R64 & 6.936118 & 80.774674 & \\
\hline R65 & 6.933977 & 80.775414 & \\
\hline R66 & 6.933444 & 80.776465 & \\
\hline R67 & 6.933636 & 80.77742 & \\
\hline R68 & 6.932507 & 80.77771 & \\
\hline R69 (DMB68) & 6.932156 & 80.778997 & \\
\hline $\mathrm{R} 70$ & 6.931197 & 80.778214 & \\
\hline R71 & 6.928183 & 80.776251 & \\
\hline $\mathrm{R} 72$ & 6.927821 & 80.7758 & \\
\hline $\mathrm{R} 73$ & 6.928396 & 80.775693 & \\
\hline R74 & 6.927927 & 80.775414 & \\
\hline R75 & 6.927607 & 80.775671 & \\
\hline R76 & 6.927287 & 80.775317 & \\
\hline R77 & 6.928096 & 80.774834 & \\
\hline R78 (DMB69) & 6.927287 & 80.776036 & \\
\hline R79 & 6.926861 & 80.776165 & \\
\hline $\mathrm{R} 80$ & 6.927713 & 80.77624 & \\
\hline R81 & 6.92718 & 80.776809 & \\
\hline $\mathrm{R} 82$ & 6.927169 & 80.777592 & \\
\hline R83 & 6.928021 & 80.777292 & \\
\hline R84 & 6.928436 & 80.777946 & \\
\hline R85 & 6.927179 & 80.777882 & \\
\hline R86 & 6.927307 & 80.774063 & \\
\hline R87 & 6.929341 & 80.7749 & \\
\hline $\mathrm{R} 88$ & 6.929607 & 80.777636 & \\
\hline R89 & 6.928435 & 80.778119 & \\
\hline R90 (DMB70) & 6.927849 & 80.777615 & \\
\hline
\end{tabular}




\begin{tabular}{|c|c|c|c|}
\hline R91 & 6.90111 & 80.802527 & \multirow{20}{*}{ Ambewela } \\
\hline R92 & 6.899555 & 80.803332 & \\
\hline R93 & 6.89915 & 80.802356 & \\
\hline R94 & 6.898479 & 80.802249 & \\
\hline R95 & 6.897712 & 80.802807 & \\
\hline R96 & 6.897446 & 80.803579 & \\
\hline R97 & 6.896019 & 80.802066 & \\
\hline R98 & 6.889798 & 80.799513 & \\
\hline R99 & 6.891205 & 80.801068 & \\
\hline R100 & 6.890555 & 80.801358 & \\
\hline R101 & 6.89079 & 80.797774 & \\
\hline R102 & 6.890588 & 80.800113 & \\
\hline R103 & 6.889789 & 80.800928 & \\
\hline R104 & 6.889074 & 80.801079 & \\
\hline R105 & 6.889362 & 80.800167 & \\
\hline R106 & 6.889936 & 80.800489 & \\
\hline R107 & 6.888626 & 80.800167 & \\
\hline R108 & 6.888551 & 80.799169 & \\
\hline R109 & 6.888157 & 80.800242 & \\
\hline R110 & 6.888338 & 80.80124 & \\
\hline R111 & 6.853043 & 80.83038 & \multirow{10}{*}{$\begin{array}{l}\text { World's end road, Horton Plains National } \\
\text { park }\end{array}$} \\
\hline R112 & 6.853032 & 80.828996 & \\
\hline R113 & 6.852467 & 80.827108 & \\
\hline R114 & 6.851881 & 80.825595 & \\
\hline R115 & 6.851498 & 80.824705 & \\
\hline R116 & 6.851466 & 80.821615 & \\
\hline R117 & 6.850124 & 80.820424 & \\
\hline R118 & 6.850177 & 80.816744 & \\
\hline R119 & 6.847812 & 80.813794 & \\
\hline R120 & 6.844275 & 80.814298 & \\
\hline R121 & 6.840089 & 80.812335 & \multirow{20}{*}{ Entrance of the Horton Plains National park } \\
\hline R122 & 6.840078 & 80.811198 & \\
\hline R123 & 6.839439 & 80.811101 & \\
\hline R124 & 6.839492 & 80.811744 & \\
\hline R125 & 6.839727 & 80.811787 & \\
\hline R126 & 6.839662 & 80.811047 & \\
\hline R127 & 6.839305 & 80.812522 & \\
\hline R128 & 6.839917 & 80.810499 & \\
\hline R129 & 6.83997 & 80.812913 & \\
\hline R130 & 6.839959 & 80.813396 & \\
\hline R131 & 6.839426 & 80.813085 & \\
\hline R132 & 6.83916 & 80.812388 & \\
\hline R133 & 6.838915 & 80.812839 & \\
\hline R134 & 6.838755 & 80.813397 & \\
\hline R135 & 6.841248 & 80.81227 & \\
\hline R136 & 6.839544 & 80.813901 & \\
\hline R137 & 6.839768 & 80.812324 & \\
\hline R138 & 6.839442 & 80.811989 & \\
\hline R139 & 6.839503 & 80.811681 & \\
\hline R140 & 6.839242 & 80.812228 & \\
\hline
\end{tabular}




\begin{tabular}{ccc}
\hline R141 (DMB61) & 6.839959 & 80.813396 \\
R142 & 6.982601 & 80.751725 \\
R143 & 6.9839 & 80.75209 \\
R144 & 6.985125 & 80.7512 \\
R145 & 6.987127 & 80.750846 \\
R146 (DMB62) & 6.993112 & 80.746855 \\
R147 & 6.994198 & 80.747745 \\
R148 & 6.995029 & 80.748571 \\
R149 & 6.995806 & 80.747831 \\
R150 & 6.995881 & 80.74679 \\
R151 & 6.997063 & 80.74487 \\
R152 & 6.99816 & 80.744484 \\
R153 & 6.999033 & 80.744012 \\
R154 & 6.999241 & 80.743282 \\
R155 & 6.998858 & 80.742826 \\
R156 & 6.998949 & 80.741388 \\
R157 (DMB63) & 7.000056 & 80.741211 \\
R158 & 7.000471 & 80.74164 \\
R159 & 7.000854 & 80.741012 \\
R160 (DMB64) & 7.00103 & 80.740771 \\
R161 & 7.00136 & 80.740326 \\
R162 & 7.001839 & 80.740278 \\
R163 (DMB65) & 7.002371 & 80.740283 \\
\hline
\end{tabular}

Table 03: The Bioclimatic variables and their parentage contribution for occurrence of the species assessed.

\begin{tabular}{lc}
\hline \multicolumn{1}{c}{ Bio Climatic Variable } & Percentage contribution (\%) \\
\hline Mean Temperature of Coldest Quarter & 24.0 \\
Mean Temperature of Warmest Quarter & 17.6 \\
Precipitation Seasonality [Coefficient of Variation] & 11.2 \\
Min Temperature of Coldest Month & 11.0 \\
Annual Mean Temperature & 10.1 \\
Precipitation of Coldest Quarter & 6.9 \\
Annual Precipitation & 4.4 \\
Max Temperature of Warmest Month & 3.3 \\
Temperature Seasonality & 2.4 \\
Precipitation of Wettest Quarter & 1.9 \\
Mean Temperature of Wettest Quarter & 1.5 \\
Precipitation of Driest Month & 1.2 \\
Precipitation of Wettest Month & 1.2 \\
Precipitation of Driest Quarter & 1.0 \\
Precipitation of Warmest Quarter & 1.0 \\
Isothermality & 0.9 \\
Temperature Annual Range & 0.4 \\
Mean Diurnal Range [Mean of monthly (max temp - min temp)] & 0.0 \\
Mean Temperature of Driest Quarter & 0.0 \\
\hline
\end{tabular}




\section{RESULTS}

\section{Phylogenetic position of Sri Lankan wild strawberries}

The phylogenetic analysis carried out in both ML and Bayesian frameworks resulted almost congruent tree topologies. The ML analysis produced a tree with higher-log-likelihood value with well-supported clades. The MCMC chains in the Bayesian analysis reached maximum convergence at initial 50,000 generations thus trees probed up to that point were discarded as burn-in. The ESS values $(<200)$ indicated the independent sampling of trees after maximum chain convergence. Thus the 50 million chain runs were enough to obtain a robust phylogeny. The 50\% majority rule consensus tree constructed in the Bayesian criteria also had well supported and well-resolved branches. Since both ML and Bayesian trees had almost similar branching patterns, we only present the ML tree with ML bootstrap values and Bayesian posterior probabilities imprinted on each node.

As depicted in previous studies (Feng et al., 2017), our phylogeny constructed for the tribe Potentilleae had three main clades separating the sub tribes Potentilla, Argentina and Fragariinae with the PP of 100 and the bs of 100 support values. The combined analysis of plastid and nuclear DNA markers also recovered the clade topology where sub tribe Potentilla clade sister to the sub tribe Argentina ( $\mathrm{bs}=99, \mathrm{PP}=96$ ) parallel with the findings of a previous study (Töpel et al., 2011). In congruence with previous studies ((Töpel et al., 2011; Feng et al., 2017), the ML tree also resolved the five lineages within subtribe Potentilla with high node support values (Figure 01). Similarly, we observed a similar branching pattern for subtribes Argentina and Fragariinae indicating the accuracy of the phylogenetic analysis. The wild strawberry samples sequenced during the present study included under Reptans clade of subtribe Potentilla with a slight divergence to Potentilla indica $(\mathrm{bs}=100, \mathrm{pp}=100)$ (Figure 01 ). The topology of $P$. indica did not recover any reciprocal monophyly between the samples collected from Nuwara-Eliya, Radella, and Mipillimana populations of the NuwaraEliya District.

\section{Predicted distribution of Sri Lankan wild strawberries}

We assessed the niche model performances using three methods. Initially, we evaluated the model performance using threshold-independent criteria, where we checked the AUC values of the ROC curves. For Auto and $\mathrm{H}$ threshold rule features, the AUC curves functioned similarly with the highest AUC value of 0.998 to the lowest AUC value of 0.995 (Figure $02 \mathrm{~A}$ and B). The optimum $\beta$ regulation parameters for these features were 0.01 and 0.1 . However, for $\mathrm{P}$ threshold rule feature, $\mathrm{H}$ feature, and $\mathrm{L}+\mathrm{Q}+\mathrm{P}$ features, the AUC was constant at 0.995 at all the regulation parameters (Figure $02 \mathrm{C}, \mathrm{D}$ and E). The AUC curves functioned similarly for $\mathrm{L}+\mathrm{Q}+\mathrm{P}+\mathrm{T}$ features and $\mathrm{L}+\mathrm{Q}+\mathrm{P}+\mathrm{T}+\mathrm{H}$ features where the highest AUC values of 0.998 to the lowest AUC value of 0.995 (Figure $02 \mathrm{~F}$ and $\mathrm{G}$ ). The optimum $\beta$ regulation parameters for these features were 0.01 and 0.1 . Next, we chose the best models which had the highest AUC values and checked the model performance by analyzing omission and commission graphs. We tested the best performance at the omission of training samples concerning the predicted omission. The best performance was observed for $\beta=0.01$ and 0.1 where the $\mathrm{L}+\mathrm{Q}+\mathrm{P}+\mathrm{T}$ and $\mathrm{L}+\mathrm{Q}+\mathrm{P}+\mathrm{T}+\mathrm{H}$ threshold features were applied. Finally, we checked the visual outputs to select the final model that best fit for our occurrence data of wild strawberries. After tuning our model, we chose the model which applied $\mathrm{L}+\mathrm{Q}+\mathrm{P}+\mathrm{T}+\mathrm{H}$ threshold features at $\beta=0.01$ (Figure 02).

According to the ENM, the most important environmental variable for species occurrence was the mean temperature of the coldest quarter ( $24 \%$ contribution). However, the mean temperature of the warmest quarter $(17.6 \%$ contribution), seasonality precipitation (11.2\% contribution), the minimum temperature at the coldest quarter (11.2\% contribution) and annual mean temperature $(10.1 \%$ contribution) were also crucial for the species occurrence. According to the ENM, the predicted distribution was restricted to Nuwara-Eliya District at an area of $166.36 \mathrm{~km}^{2}$ (Figure 03A, B, C, D). The predicted distribution also overlaps with our sampling scheme, where we found a restricted occurrence 
of wild strawberry in the Nuwara-Eliya District (Figure 03. E and F). Also, the species occurs in Sri Lanka in the altitude of $1546 \mathrm{~m}-2524 \mathrm{~m}$ (Figure 03G).

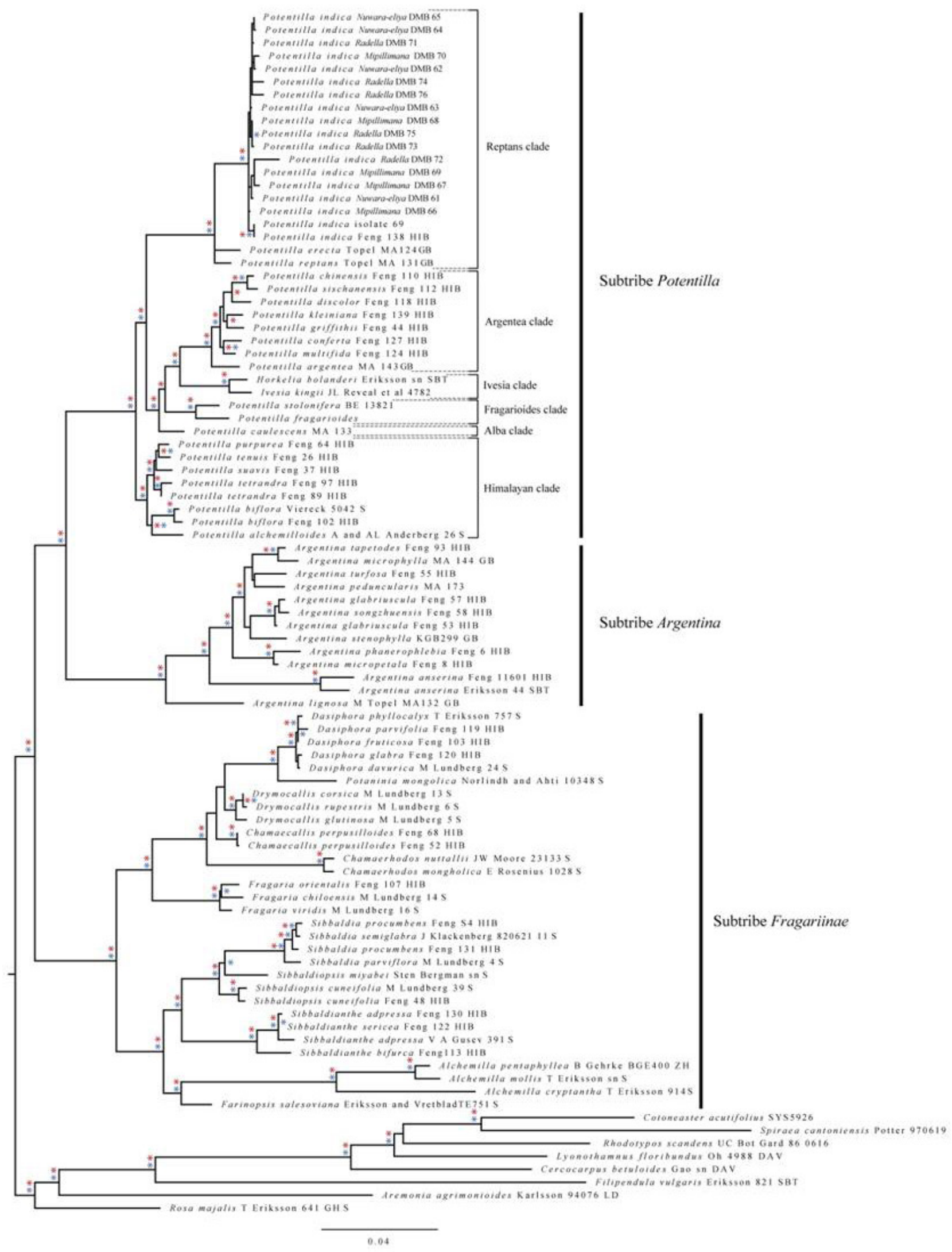

Figure 01: The majority rule consensus tree constructed in Maximum likelihood framework for combined datasets of ITS and $\operatorname{trn} L-F$. The red * indicates the nodes that are having a posterior probability higher than 90 . The blue * indicates the nodes that are having a bootstrap value higher than 80 . The subtribes and lineages are given in front of the respective clade. 

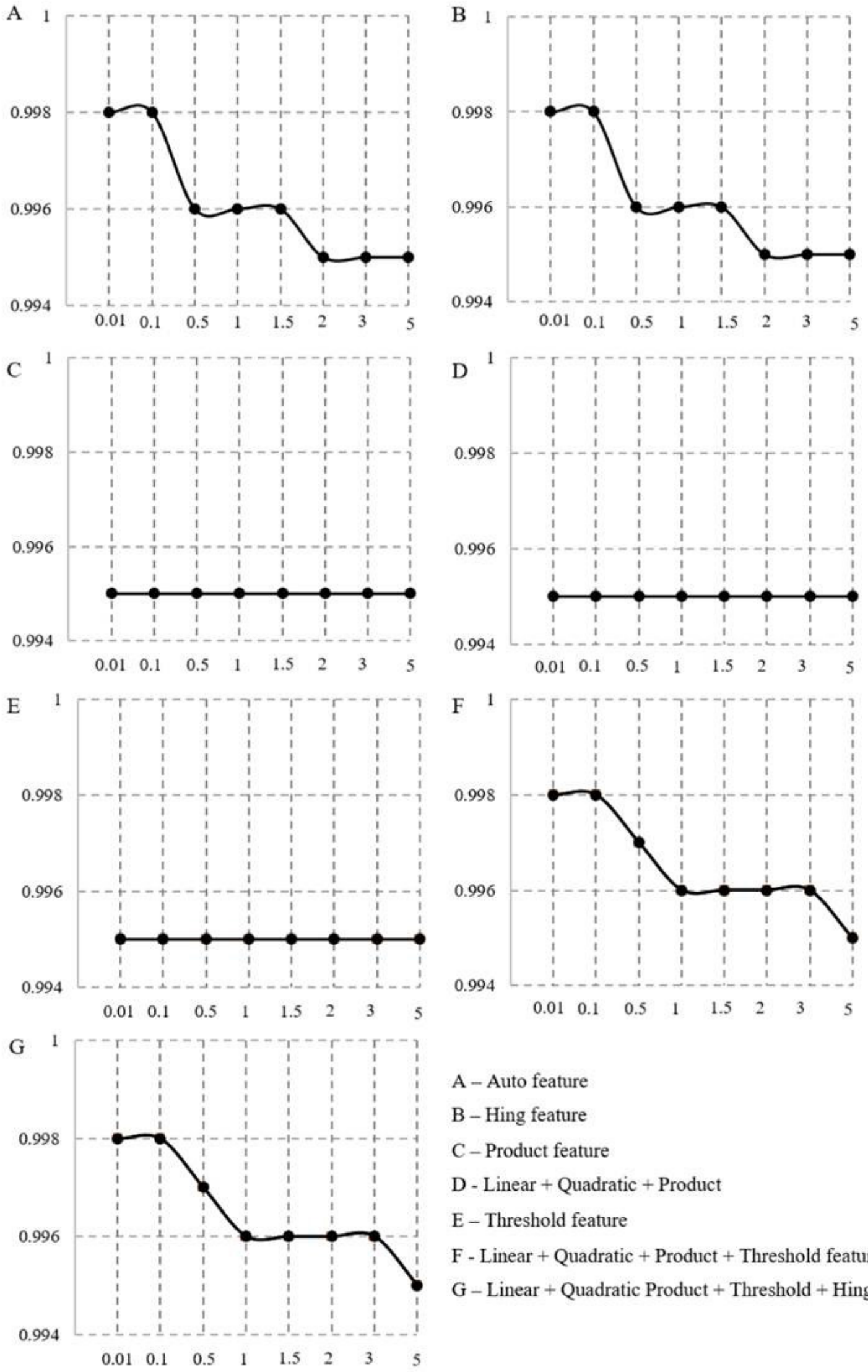
A - Auto feature
B - Hing feature
$\mathrm{C}$ - Product feature
D - Linear + Quadratic + Product
E - Threshold feature
F - Linear + Quadratic + Product + Threshold features
$\mathrm{G}-$ Linear + Quadratic Product + Threshold + Hing feature

Figure 02: The Receiver Operation Curves (ROC) showing the behavior of AUC values with respect to the $\beta$ regulation parameter under different threshold rules. The $X$ axis represent the AUC value and the $Y$ axis represent the value for the $\beta$ parameter. 


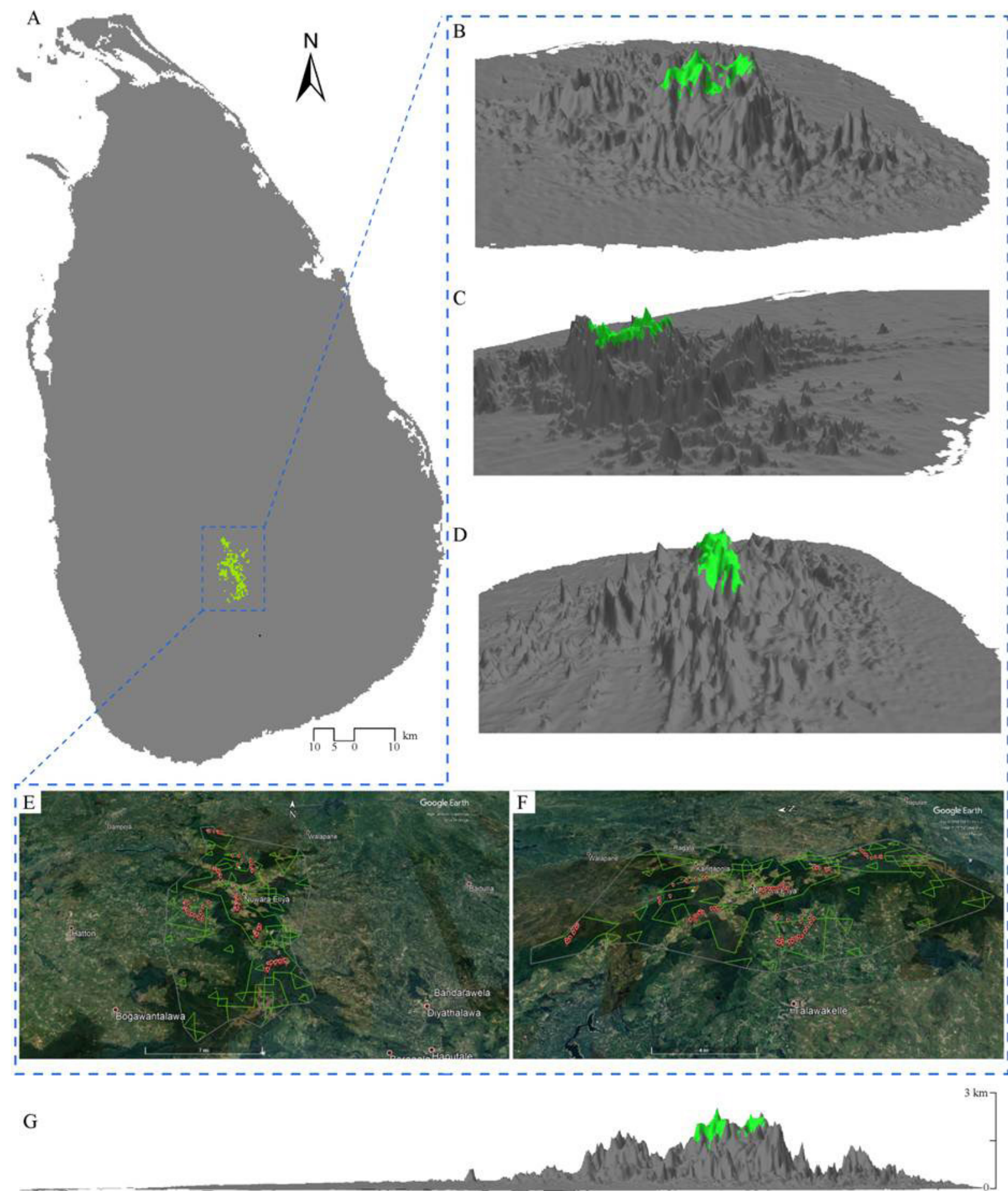

Figure 03: The predicted distribution of the study species. A: The expected distribution overlaid to map of Sri Lanka. B: 3-D visualization of Sri Lanka showing the predicted niche from the western slope of Central hills. C: 3-D visualization of Sri Lanka showing the predicted niche from Northeastern slope of Central hills. D: 3-D visualization of Sri Lanka showing the predicted niche from the southern slope of Central hills. $E$ and $F$ : The satellite image of the area of the predicted niche showing geographical features, predicted niche (green lines), the overall area of the predicted niche (grey line), and the presence-localities used for distribution prediction. G: Elevational profile of Sri Lanka showing the climatic envelop of $P$. indica. 


\section{DISCUSSION}

In the present study, we aim to identify the phylogenetic position of wild strawberry (Indian strawberry or mock strawberry) through a phylogenetic analysis of nuclear and plastid markers. We also carried out an extensive sampling scheme and a distribution prediction using niche model approach to re-locate the full distribution of wild strawberry. A combined phylogeny of trnL-trnF and ITS markers resolved congruent to previously published data (Feng et al., 2017; Dobeš and Paule, 2010; Töpel et al., 2011). Our adapted phylogeny from Feng et al. (2017) also resolved some of the polytomic positions reported in previous studies. The employment of data partition matrices to capture the differential evolutionary processes of chloroplast and nuclear markers resulted more robust phylogeny with higher node support values. The phylogenetic positions of the samples sequenced during the present study cladded under sub-tribe Potentilla, lineage Reptans and the clade (species) Potentilla indica. Although this species is previously named as Duchesnea indica in the Sri Lanka descriptors (Wadhwa et al., 2000), now it is possible to revise the generic name as Potentilla indica as observed in our phylogeny. The $P$. indica (nov.) inhabited in Sri Lanka shows a uniform genetic structure for the $P$. indica sequences reported in previous studies. Although we sequenced samples from three distinct populations, no variations were identified between populations.

Since $P$. indica is a runnering plant, it is possible to have less variation within a population. However, due to the polyploid nature of Potentilla, there could be variations among the plants that arise from seeds. The clade structure with slight divergence must have been occurred due to the polyploid genetics. We identified a high gene flow among the populations of $P$. indica in Radella, Nuwara-Eliya, and Mipillimana. We believe that the dispersal of $P$. indica is mainly occurring through birds maintaining higher gene flow among the populations. In our sampling, we found wild strawberries abundantly in Nuwara-Eliya district in disturbed and opened habitats with high exposure to the sun. Despite higher abundance in Nuwara-Eliya district, we did not find wild strawberry in other higher elevations, mountain ranges such as Knuckles and Rakwana hills. Moreover, our niche model analysis predicted a similar distribution pattern in line with our field sampling (Figure 03E and 03F). The predicted distribution of $P$. indica is restricted to the about $166.36 \mathrm{~km}^{2}$ area in Nuwara-Eliya District (Figure 03A). Our predicted distribution also shows the occurrence of $P$. indica exclusively restricted to high altitudes (Figure 03B, C, and D), starting from $1546 \mathrm{~m}$ to $2524 \mathrm{~m}$ (Figure 03G). Thus it is apparent that $P$. indica in Sri Lanka is highly restricted by a climatically enveloped structure. According to our entropy modeling, the most important climatic parameter for the occurrence of $P$. indica is the mean temperature of the coldest quarter (24\% contribution) (Table 03$)$.

According to our results, it is apparent that the distribution of $P$. indica in Sri Lanka is directly correlated with the temperature variation in highland and seasonality of the precipitation. Although $P$. indica has the capability of distributing into other mountain ranges, due to the climatic envelope, the growth and dispersal are highly governed by the climatic factors. Thus we identified a mountain isolation in which habitat shrinkage could easily be resulted with the global climate change. With the rising environmental pollution, one of the most common problems for floral and faunal populations is the mountain isolation (Busby, 1988; Hamilton, 1995; Foste, 2001; Peñuelas and Boada, 2003; Feng et al., 2016). With the drastic increment of global warming, the floral and faunal species that prefer the coldest environmental conditions tend to confine into higher altitudes. Since the $P$. indica germplasm in Sri Lanka is restricted with climatic envelop, we believe it is currently at a threat and requires a conservation priority demanding urgent conservation measures.

\section{CONCLUSIONS}

In the present study, we carried out a systematic revision and species distribution prediction to identify the conservation needs of wild (Indian or mock) strawberries inhabited in Sri Lanka. Through a phylogenetic analysis, we revealed that Sri Lankan wild strawberry germplasm clades with subtribe: Potentilla, clade: Reptans 
$(\mathrm{PP}=100, \mathrm{bs}=100)$. The shallow divergence of the study species with Potentilla indica species indicates the need of taxonomic repositioning of this genus. Thus we reposition the genus of study species from Duchesnea to Potentilla and hereafter name wild strawberries in Sri Lanka as $P$. indica. After smoothening the maximum entropy model, we chose the model which applied $\mathrm{L}+\mathrm{Q}+\mathrm{P}+\mathrm{T}+\mathrm{H}$ threshold features at $\beta=0.01$ that had a high AUC value (0.998). The predicted niche indicated that $P$. indica (nov.) has a highly restricted distribution. The $P$. indica was only dispersed in Nuwara-Eliya District over 166.36 $\mathrm{km}^{2}$ of the area found in the altitude range of 1546 $\mathrm{m}-2524 \mathrm{~m}$. Since the $P$. indica germplasm in Sri Lanka is restricted within a climatic envelop, we believe that it is under threat and requires a conservation priority.

\section{ACKNOWLEDGEMENTS}

Authors wish to thank site-owners of the plants for granting permission to access and collect samples.

\section{Data Availability Statement}

The nucleotide dataset generated during and/or analyzed during the current study are available in the GenBank, and https://www.ncbi.nlm.nih. gov/nuccore (ITS: MK587724-MK587739; trnLtrnF: MN605458-MK605473; Table 01).

The other datasets generated during and/or analyzed during the current study are available from the corresponding author on reasonable request.

\section{REFERENCES}

Anderson, R.P. and Gonzalez Jr, I. (2011) Species-specific tuning increases robustness to sampling bias in models of species distributions: an implementation with Maxent. Ecological Modelling. 10(15), pp.2796-2811. DOI: https://doi.org/10.1016/j.ecolmodel.2011.04.011.

Andrews, H.C. (1807). Botanists repository. London. 7pp.

Busby, J.R. (1988). Potential impacts of climate change on Australia's flora and fauna. Commonwealth Scientific and Industrial Research Organisation: MelbourneFL: USA.

Cavanaugh, J.E. (1997) Unifying the derivations for the Akaike and corrected Akaike information criteria. Statistics and Probability Letters. 33(2), pp.8-201. DOI: https://doi.org/10.1016/ S0167-7152(96)00128-9.

Dobeš, C. and Paule, J. (2010). A comprehensive chloroplast DNA-based phylogeny of the genus Potentilla (Rosaceae): implications for its geographic origin, phylogeography and generic circumscription. Molecular Phylogenetics and Evolution. 56, pp.156-175. DOI: https://doi. org/10.1016/j.ympev.2010.03.005.

Elith, J., Graham, C.H., Anderson, R.P., Dudík, M., Ferrier, S., Guisan, A., Hijmans, R.J., Huettmann, F., Leathwick, J.R., Lehmann, A. and Li, J., Overton, Peterson, A.T, Phillips, S.J., Richardson K.S., Scachetti-Pereira, R., Schapire, R.E., Soberón, J., Williams, S., Wisz, M.S. and Zimmermannet, N.E. (2006). Novel methods improve prediction of species' distributions from occurrence data. Ecography. 29, pp.129-151. DOI/; https://doi.org/10.1111/j.2006.0906-7590.04596.x.

Elith, J., Phillips, S.J., Hastie, T., Dudík, M., Chee, Y.E. and Yates, C.J. (2011). A statistical explanation of MaxEnt for ecologists, Diversity and Distributions. 17(1), pp.43-57. DOI: https://doi. org/10.1111/j.1472-4642.2010.00725.x.

Eriksson, T., Donoghue, M.J. and Hibbs, M.S. (1998). Phylogenetic analysis of Potentilla using DNA sequences of nuclear ribosomal internal transcribed spacers (ITS), and implications for the classification of Rosoideae (Rosaceae). Plant Systematics and Evolution. 211, pp.155-179. DOI: https://doi.org/10.1007/BF00985357. 
Eriksson, T., Hibbs, M.S., Yoder, A.D., Delwiche, C.F. and Donoghue, M.J. (2003). The phylogeny of Rosoideae (Rosaceae) based on sequences of the internal transcribed spacers (ITS) of nuclear ribosomal DNA and the trnL/F region of chloroplast DNA. International Journal of Plant Sciences. 164, pp.197-211. DOI: https://doi.org/10.1086/346163.

Faith, D.P. (1996). Conservation priorities and phylogenetic pattern. Conservation Biology. 10, pp.1286-1289. DOI: https://doi.org/10.1046/j.1523-1739.1996.10041286.x.

Feng, B., Zhao, Q., Xu, J., Qin, J. and Yang, Z.L. (2016). Drainage isolation and climate change-driven population expansion shape the genetic structures of Tuber indicum complex in the Hengduan Mountains region. Scientific Reports. 6, pp.21811. DOI: https://doi.org/10.1038/srep21811.

Feng, T., Moore, M.J., Yan M.H., Sun, Y.X., Zhang, H.J., Meng, A.P., Li, X.D., Jian, S.G., Li, J.Q. and Wang, H.C. (2017). Phylogenetic study of the tribe Potentilleae (Rosaceae), with further insight into the disintegration of Sibbaldia. Journal of Systematics and Evolution. 55, pp.177191. DOI: https://doi.org/10.1111/jse.12243.

Focke, W.O. (1888).Rosaceae in Engler, A. Natürliche Pflanzenfamilien, Leipzig.

Foster, P. (2001). The potential negative impacts of global climate change on tropical montane cloud forests. Earth-Science Reviews. 55(1-2), pp.73-106. DOI: https://doi.org/10.1016/S00128252(01)00056-3.

Frandsen, P.B., Calcott, B., Mayer C. and Lanfear, R. (2015). Automatic selection of partitioning schemes for phylogenetic analyses using iterative k-means clustering of site rates. $B M C$ Evolutionary Biology. 15(1), pp13. DOI: https://doi.org/10.1186/s12862-015-0283-7.

Geneletti, D.A. (2004). GIS-based decision support system to identify nature conservation priorities in an alpine valley. Land Use Policy. 21, 149-160. DOI: https://doi.org/10.1016/j. landusepol.2003.09.005.

Hamilton, L.S. (1995). Mountain cloud forest conservation and research: a synopsis. Mountain Research and Development. 1, pp.259-266. DOI: 10.2307/3673933

Hernandez, P.A., Graham, C.H., Master, L.L. and Albert, D.L. (2006). The effect of sample size and species characteristics on performance of different species distribution modeling methods. Ecography. 29, pp.773-785. DOI: https://doi.org/10.1111/j.0906-7590.2006.04700.x.

Hortal, J., Jiménez-Valverde, A., Gómez, J.F., Lobo, J.M. and Baselga, A. (2008). Historical bias in biodiversity inventories affects the observed environmental niche of the species. Oikos. 117(6), pp.847-858. DOI: https://doi.org/10.1016/j.ecolmodel.2005.03.026.

Huelsenbeck, J.P. and Ronquist, F. (2001). MRBAYES: Bayesian inference of phylogenetic trees. Bioinformatics, 17, pp.754-755. DOI: 10.1093/bioinformatics/17.8.754

Kozak, K.H., Graham, C.H. and Wiens, J.J. (2008). Integrating GIS-based environmental data into evolutionary biology. Trends in Ecology and Evolution. 23:, pp.41-48. DOI: https://doi. org/10.1016/j.tree.2008.02.001.

Kumar, S., Stecher, G. and Tamura, K. (2016). MEGA7: molecular evolutionary genetics analysis version 7.0 for bigger datasets. Molecular Biology and Evolution. 33(7), pp.1870-1874. DOI: https://doi.org/10.1093/molbev/msw054. 
Lanfear, R., Calcott, B., Kainer, D., Mayer C. and Stamatakis, A. (2014). Selecting optimal partitioning schemes for phylogenomic datasets. BMC Evolutionary Biology. 14(1), pp.82. DOI: https://doi. org/10.1186/1471-2148-14-82.

Lanfear, R., Frandsen, PB., Wright, AM., Senfeld, T. and Calcott, B. (2016). PartitionFinder 2: new methods for selecting partitioned models of evolution for molecular and morphological phylogenetic analyses. Molecular Biology and Evolution. 34(3), pp.772-773. DOI: https://doi. org/10.1093/molbev/msw260.

Miller, M.A., Pfeiffer, W. and Schwartz, T. (2010). Creating the CIPRES Science Gateway for inference of large phylogenetic trees. In: gateway computing environments workshop (GCE). $1-8$.

Peñuelas, J. and Boada, M. (2003). A global change induced biome shift in the Montseny mountains (NE Spain). Global Change Biology. 9(2), pp.131-140. DOI: https://doi.org/10.1046/j.13652486.2003.00566.x.

Peterson, A.T. (2003). Predicting the geography of species' invasions via ecological niche modeling. The Quarterly Review of Biology. 78, pp.419-433. DOI: https://doi.org/10.1086/378926.

Peterson, A.T., Soberón, J., Pearson, R.G., Anderson, R.P., Martínez-Meyer, E., Nakamura M. and Araújo, M.B. (2011). Ecological niches and geographic distributi.ons. Princeton University Press: Princeton.

Phillips, S.J. and Dudík, M. (2008). Modeling of species distributions with Maxent: new extensions and a comprehensive evaluation. Ecography. 31, 161-175. DOI: https://doi.org/10.1111/j.09067590.2008.5203.x.

Phillips, S.J., Anderson, R.P. and Schapire, R.E. (2006). Maximum entropy modeling of species geographic distributions. Ecological Modelling. 190(3-4), pp.231-259.

Phillips, SJ., Dudík, M. and Schapire, R.E. (2004). A maximum entropy approach to species distribution modeling. In Proceedings of the twenty-first international conference on Machine learning. DOI: $10.1145 / 1015330.1015412$.

Planet, P.J. (2006). Tree disagreement: measuring and testing incongruence in phylogenies, Journal of Biomedical Informatics. 39(1), pp.86-102. DOI: https://doi.org/10.1016/j.jbi.2005.08.008.

Potter, D., Still, S.M., Grebenc, T., Ballian, D., Božič, G., Franjiæ, J. and Kraigher, H. (2007). Phylogenetic relationships in tribe Spiraeeae (Rosaceae) inferred from nucleotide sequence data. Plant Systematics and Evolution. 266, pp.105-118. DOI: https://doi.org/10.1007/s00606007-0544-z.

Rambaut, A. and Drummond, A. (2007). Tracerv1. 4. http://beast. bio. ed. ac. uk/Tracer. 15.12.2018.

Rambaut, A. (2014). FigTree, a graphical viewer of a phylogenetic trees. http://tree. bio. ed.ac. uk/ software/figtree. 20.12.2018.

Senavirathna, H.M.T.N., Ranaweera, L.T., Mudannayake, M.M.A.W.P., Nawanjana, P.W.I., Wijesundara, W.M.D.A., Jayarathne, H.S.M., Ratnasuriya, M.A.P., Weebadde, C.K. and Sooriyapathirana, S.D.S.S. (2020). Assessment of the taxonomic status of the members of genus Artocarpus (Moraceae) in Sri Lanka. Genetic Resources and Crop Evolution. 67, pp. 1163-1179. DOI: https://doi.org/10.1007/s10722-020-00902-x 
Senevirathne, G., Samarawickrama, V.A., Wijayathilaka, N., Manamendra-Arachchi, K., Bowatte, G. and Samarawickrama, D.R. (2018). A new frog species from rapidly dwindling cloud forest streams of Sri Lanka-Lankanectes pera (Anura,Nyctibatrachidae), Zootaxa. 23, pp.519-538. DOI: http://dx.doi.org/10.11646/zootaxa.4461.4.4.

Stamatakis, A. (2006). RAxML-VI-HPC: maximum likelihood-based phylogenetic analyses with thousands of taxa and mixed models. Bioinformatics. 22, pp.2688-2690. DOI: https://doi. org/10.1093/bioinformatics/btl446.

Stamatakis, A., Hoover P. and Rougemont, J. (2008). A rapid bootstrap algorithm for the RAxML web servers. Systematic Biology. 57, pp.758-771.DOI: https://doi.org/10.1080/10635150802429642.

Taberlet, P., Gielly, L., Pautou, G. and Bouvet, J. (1991). Universal primers for amplification of three non-coding regions of chloroplast DNA. Plant Molecular Biology. 17(5), pp.1105-1109. DOI: https://doi.org/10.1007/BF00037152.

Töpel, M., Lundberg, M., Eriksson, T. and Eriksen, B. (2011). Molecular data and ploidal levels indicate several putative allopolyploidization events in the genus Potentilla (Rosaceae). PLoS Currents. 3. DOI: 10.1371/currents.RRN1237.

Wadhwa, B.M. (2000). Duchesnea, In: A revised handbook to the Flora of Ceylon. (Dassanayake, M.D. and Clayton, W.D. Eds.). Oxford and IBH Publishing Co. Pvt. Ltd, New Delhi, India. 361- 364.

White, T.J., Bruns, T., Lee, S. and Taylor, J.W. (1990) Amplification and direct sequencing of fungal ribosomal RNA genes for phylogenetics. In: PCR Protocols: A Guide to Methods and Applications (Innis, M.A., Gelfand, D.H., Sninsky, J.J. and White, T.J. Eds). San Diego (CA): Academic Press. 315-322.

Wiens, J.J. and Graham, C.H. (2005). Niche conservatism: integrating evolution, ecology, and conservation biology. Annual Review of Ecology, Evolution, and Systematics. 36, 519-539. DOI: https://doi.org/10.1146/annurev.ecolsys.36.102803.095431.

Wijayathilaka, N., Senevirathne, G., Bandara, C., Rajapakse, S., Pethiyagoda, R and Meegaskumbura, M. (2018) Integrating bioacoustics, DNA barcoding and niche modeling for frog conservationThe threatened balloon frogs of Sri Lanka. Global Ecology and Conservation. 1, pp.16. DOI: https://doi.org/10.1016/j.gecco.2018.e00496.

Wisz, M.S., Hijmans, R.J., Li, J., Peterson, A.T., Graham, C.H. and Guisan, A. (2008). NCEAS Predicting Species Distributions Working Group, Effects of sample size on the performance of species distribution models. Diversity and Distributions. 14, pp.763-773. DOI: https://doi. org/10.1111/j.1472-4642.2008.00482.x.

Wolf, F.T. (1908). Monographie der Gattung Potentilla. Bibliotheca Botanica. 16, pp.1-714. 